\title{
Transvenous pacing after tricuspid valve replacement with a Starr-Edwards prosthesis
}

\author{
Clive Layton and Roger Boyle \\ From the Cardiac Department, The London Hospital, Whitechapel, London
}

Transvenous right ventricular pacing was performed in a patient with a Starr-Edwards tricuspid valve prosthesis to control a life-threatening dysrhythmia. Apical siting of the electrode was associated with severe valvar dysfunction but pacing was established from the ventricular outflow tract at the expense of tricuspid regurgitation. Despite considerable disturbance of the catheter position with each systole, stable pacing was maintained for a period of 60 hours. In an emergency situation the presence of a prosthetic tricuspid valve is no bar to transvenous right ventricular pacing.

Artificial pacing is an accepted form of treatment for a wide range of disturbances of cardiac rhythm (Sowton, Leatham, and Carson, 1964; Cohen, Kahn, and Donoso, 1967). Stimulation of the myocardium through a right ventricular endocardial electrode is the method of choice in most cases and particularly when the need to establish pacing is urgent. The positioning of such an electrode can be rapidly performed under fluoroscopic control and is even possible without radiographic assistance by monitoring the course of the electrode from a continuous recording of the electrocardiogram from its tip as it passes through the right atrium and ventricle (Kimball and Killip, 1965; Chatterjee et al., 1969). Successful right ventricular pacing has been reported in patients with a prosthetic tricuspid disc valve (Maramba et al., I97I) but not in the presence of a Starr-Edwards prosthesis, the most commonly used artificial valve. We therefore wish to report a case of endocardial right ventricular pacing in a patient with a tricuspid Starr-Edwards prosthetic valve in the control of a life-threatening dysrhythmia.

\section{Case report}

A 23-year-old woman was first seen at this hospital in I940 with a history of slight shortness of breath on exertion. A diagnosis of mitral stenosis was made and the patient was not seen again until 1952. At this time there had been an increase in exertional dyspnoea but no other symptoms. On examination there were signs of severe mitral stenosis. The chest $x$-ray showed enlargement of the left atrium and main pulmonary artery and the electrocardiogram showed no abnormality other than a P mitrale.
A closed mitral valvotomy was performed with considerable relief of symptoms, but a pansystolic murmur was heard postoperatively which was thought to represent mitral regurgitation. The next 16 years were uneventful apart from the development of atrial fibrillation in 1958 and a possible small pulmonary embolism in 1966 while on anticoagulant therapy.

In 1968 the patient returned complaining of a rapid increase in exertional dyspnoea, nausea, and abdominal distension. The physical signs were of gross congestive cardiac failure and tricuspid regurgitation. The electrocardiogram showed atrial fibrillation, right ventricular hypertrophy, and ST segment changes compatible with the effects of digitalization. The chest $x$-ray again showed left atrial and pulmonary artery enlargement but also pulmonary oedema. After a period of treatment with rest and diuretics during which the jugular venous pressure fell to normal, cardiac catheterization was performed, and confirmed the presence of severe mitral stenosis and regurgitation. At the end of 1968 mitral valve replacement was performed by Mr. E. J. M. Weaver using an aortic valve homograft. Slight tricuspid regurgitation was noted at operation, but the postoperative course was uneventful and the initial result good, with improvement of exercise tolerance.

The patient was readmitted to hospital in 1972 with rapid deterioration of symptoms, and once again signs of severe congestive cardiac failure were present. In addition, a loud pansystolic murmur was heard which increased on inspiration. A systolic wave was again present in the jugular venous pulse. Cardiac catheterization was repeated at this time. Pressure recordings were as follows: right atrium (mean) $18 \mathrm{mmHg}$, with a systolic wave of $26 \mathrm{mmHg}$, right ventricle $60 / 5 \mathrm{mmHg}$, pulmonary artery $60 / 30 \mathrm{mmHg}$, left ventricle I I0/5 $\mathrm{mmHg}$ (end-diastolic pressure $16 \mathrm{mmHg}$ ), pulmonary wedge pressure (mean) $30 \mathrm{mmHg}$, with a systolic wave of $50 \mathrm{mmHg}$. A left ventricular cineangiogram showed 
grade $3 / 4$ mitral regurgitation through the homograft valve.

In June 1972 operation was again performed by $\mathrm{Mr}$. Weaver under cardiopulmonary bypass. One cusp of the aortic homograft was found to have prolapsed and the valve therefore removed and replaced with a $27 \mathrm{M}$ Bjork $-y$ valve. Severe tricuspid regurgitation was al thed and this valve was therefore replaced with a model 6120 32 Starr-Edwards prosthesis. The postoperative course was complicated by recurrent episodes of asystole and ventricular fibrillation which were controlled by artificial pacing using temporary epicardial electrodes applied at the time of operation. After ro days a stable rhythm was restored with atrial fibrillation and a controlled ventricular rate. The patient was discharged from hospital five weeks after operation. Digoxin and diuretics were continued. Subsequently she made good progress again with considerable symptomatic relief. However, I I weeks after operation the patient was found collapsed in her home with no peripheral pulse or spontaneous respiration. External cardiac massage was performed with return of the pulse and consciousness. On arrival at the hospital the heart rhythm was chaotic (Fig. I), with periods of bradycardia with atrial fibrillation, ectopic beats, ventricular tachycardia, ventricular fibrillation, and asystole. Investigations performed on admission were as follows: $\mathrm{Hb}$ I $\cdot 4 \mathrm{~g} / \mathrm{roO} \mathrm{ml}$,

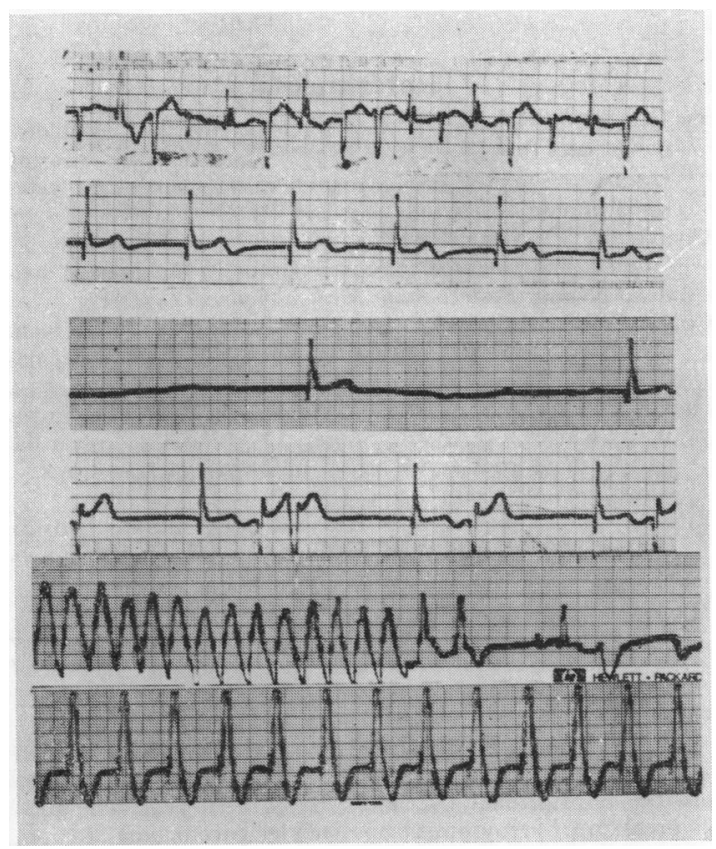

FIG. I Electrocardiographic monitoring strips showing frequent ventricular ectopic beats, atrioventricular junctional rhythm, atrial fibrillation with conspicuous bradycardia, ventricular tachycardia, and (bottom strip) stable ventricular pacing.
PCV 36.6 per cent, white cell count I I,600 $\mathrm{mm}^{3}$, with a normal differential count. Plasma sodium I34 mEq/1., potassium $2.8 \mathrm{mEq} / \mathrm{l}$., chloride $93 \mathrm{mEq} / \mathrm{l}$., and bicarbonate $30 \mathrm{mEq} / \mathrm{l}$. Urea $27 \mathrm{mg} / 100 \mathrm{ml}$. Prothrombin ratio I.3: I. Plasma digoxin level within the therapeutic range. Partial control of the dysrhythmia was obtained with intermittent lignocaine and isoprenaline, but despite rapid correction of the hypokalaemia the dysrhythmia persisted and a series of cardiac arrests occurred requiring defibrillation on three occasions. In view of the lifethreatening nature of the dysrhythmia it was decided to attempt artificial pacing. A USCI electrode (type $565 \mathrm{I}-\mathrm{S}$ ) was introduced through an antecubital vein and manipulated into the coronary sinus under fluoroscopic control. Though a variety of positions within the coronary sinus were tested, it was not possible to establish ventricular pacing from any of these sites. The electrode was therefore passed across the Starr-Edwards tricuspid valve towards the right ventricular apex. This resulted in obvious interference with movement of the ball of the prosthesis visible with the image intensifier. The electrode was immediately withdrawn and then again advanced across the valve but this time towards the outflow tract of the right ventricle. Movement of the ball continued with the electrode in this position (Fig. 2a) and stable ventricular pacing was established with a minimum stimulus of 0.6 volts with a duration of 2 milliseconds. During systole the ball of the Starr-Edwards valve was forced back, impinging on the electrode and causing the tip to dip down towards the apex of the right ventricle (Fig. 2b). Despite this instability of the electrode position, there was no loss of pacing which was continued in the ventricular inhibited mode at a rate of 90 beats per minute in order to suppress any ventricular ectopic activity. Pacing was continued for a period of 60 hours during which no further dysrhythmia occurred. However, the presence of the pacing electrode resulted in important disturbance of the function of the prosthetic valve with severe tricuspid regurgitation, the mean right atrial pressure being $45 \mathrm{mmHg}$ at this time. At the end of this period a Medtronic epicardial demand pacemaker (model 5942) was implanted and the transvenous electrode removed. There was an immediate fall in the right atrial pressure to $25 \mathrm{mmHg}$. The subsequent course was one of steady progress and at the time of discharge from hospital four weeks later the jugular venous pressure was no longer raised and exercise tolerance was much improved. Examination of the electrode did not reveal any signs of wear and in view of the successful outcome it has not been possible to examine the valve prosthesis for evidence of mechanical damage.

\section{Discussion}

Artificial pacing is widely employed in the treatment of both bradycardias and tachycardias (Rokseth et al., I970) and has greatly improved the prognosis of several types of dysrhythmia, in particular complete atrioventricular block. Pacing has also been shown to be of benefit in the management of dysrhythmias following open heart surgery (Harris et al., I968), and it is now routine procedure for tem- 
porary epicardial electrodes to be applied at the end of any corrective operation. Alternatively an endocardial electrode may be inserted transvenously if required or if the epicardial system should fail. Endocardial pacing has been performed in this way in patients after such procedures as aortic valve replacement and total correction of Fallot's tetralogy. Dysrhythmias encountered after prosthetic tricuspid valve replacement present special problems as it has not been thought that endocardial pacing could be performed successfully in view of the lack of stability of the electrode within the right ventricle and the possibility of serious interference with the function of the prosthetic valve. Maramba et al. (I97I) have described successful pacing in two patients with tricuspid disc valves without significant haemodynamic impairment but in the present case severe tricuspid regurgitation resulted due to interference with the movement of the ball within the cage of the Starr-Edwards valve. This resolved immediately the electrode was withdrawn.

Manipulation of the electrode across the prosthetic valve was achieved without any difficulty which is in accord with experience in traversing similar valves both in the aortic and tricuspid position (Bristow et al., 1966). The presence of a Starr-Edwards prosthetic tricuspid valve is therefore not a contraindication to right ventricular endocardial pacing, but in view of the severe haemodynamic disturbance which may result the procedure should be reserved for emergency use only and the electrode withdrawn at the earliest opportunity.
This patient was under the care of Dr. Wallace Brigden and Mr. E. J. M. Weaver; we are grateful to them for their advice in the preparation of this report.

\section{References}

Bristow, J. D., Kloster, F. E., Herr, R., Starr, A., McCord, C. W., and Griswold, H. E. (1966). Cardiac catheterization studies after combined tricuspid, mitral, and aortic valve replacement. Circulation, 34, 437.

Chatterjee, K., Sutton, R., Layton, C. A., and Edwards, A. (1969). The cavity electrocardiogram in emergency artificial pacing. Postgraduate Medical fournal, 45, 713.

Cohen, H. E., Kahn, M., and Donoso, E. (I967). Treatment of supraventricular tachycardias with catheter and permanent pacemakers. American Fournal of Cardiology, 20, 735.

Harris, P. D., Malm, J. R., Bowman, F. O., Jr., Hoffman, B. F., Kaiser, G. A., and Singer, D. H. (1968). Epicardial pacing to control arrhythmias following cardiac surgery. Circulation, 37, Suppl. 2, 178.

Kimball, J. T., and Killip, T. (1965). A simple bedside method for transvenous intracardiac pacing. American Heart fournal, 70, 35.

Maramba, L. C., Hildner, F. J., Greenberg, J. J., and Samet, P. (197I). Temporary pervenous pacing catheter insertion through a tricuspid prosthetic valve. American fournal of Cardiology, 27, 224.

Rokseth, R., Hatle, L., Gedde-Dahl, D., and Foss, P. O. (1970). Pacemaker therapy in sino-atrial block complicated by paroxysmal tachycardia. British Heart fournal, 32, 93.

Sowton, E., Leatham, A., and Carson, P. (I964). The suppression of arrhythmias by artificial pacemaking. Lancet, 2, 1098 .

Requests for reprints to Dr. C. A. Layton, Cardiac Department, The London Hospital, Whitechapel, London EI IBB. 